\title{
Um Sistema de Controle Neuro-Fog para Infraestruturas Residenciais via Objetos Inteligentes
}

\author{
Geraldo P. R. Filho ${ }^{1}$, José R. Torres Neto ${ }^{1}$, Alan Valejo ${ }^{1}$ \\ Rodolfo I. Meneguette ${ }^{2}$, Leandro A. Villas ${ }^{3}$, Jó Ueyama ${ }^{1}$ \\ ${ }^{1}$ Instituto de Ciência Matemáticas e de Computação - ICMC \\ Universidade de São Paulo - USP \\ ${ }^{2}$ Instituto Federal de São Paulo - IFSP \\ ${ }^{3}$ Instituto de Computação - Universidade Estadual de Campinas - UNICAMP \\ Campinas - SP - Brasil \\ \{geraldop, alan, joueyama\}@icmc.usp.br, jrtorresneto@usp.br \\ meneguettedifsp.edu.br, leandroeic.unicamp.br
}

\begin{abstract}
In recent years, the use of the fog computing paradigm is increasingly present in studies and services offered by a smart city, as the intelligent home systems which are one of the services to be highlighted. However, such paradigm brings two major challenges within the context of intelligent homes: how to extract the data that will be used in the decision-making process efficiently, and how to enable interoperability among the devices. Thus, this work proposes ImPeRIum, an intelligent decision system which forms a fog computational environment to manage the applications of the residence. ImPeRIum was evaluated in both the simulated environment and the real environment. When compared with other works in literature, ImPeRIum showed an advance in the state of the art and It obtained three promising results: (i) high hit rate with a low delay in the decision-making process; (ii) efficient in the information dissemination with a low overhead in infrastructure; and (iii) robustness in processing with a low energy consumption.
\end{abstract}

Resumo. Nos últimos anos, o uso do paradigma de fog computing está cada vez mais presente em estudos e serviços oferecidos por uma cidade inteligente, sendo os sistemas residenciais inteligentes um dos serviços a ser destacado. Entretanto, tal paradigma traz dois grandes desafios dentro do contexto de casas inteligentes: como extrair de forma eficiente os dados que serão utilizados no processo de decisão e como permitir a interoperabilidade entre os dispositivos. Com isso, este trabalho propõe o ImPeRIum, um sistema de decisão inteligente que forma um ambiente computacional de fog para gerenciar as aplicações da residência. O ImPeRIum foi avaliado tanto em um ambiente simulado quanto em um ambiente real. Quando comparado com outros trabalhos da literatura, o ImPeRIum mostrou avançar o estado da arte por obter três resultados promissores: (i) alta taxa de acertos com baixo atraso no processo de tomada de decisão; (ii) eficiência na disseminação da informação com baixo overhead na infraestrutura de comunicação; e (iii) robustez no processamento com baixo consumo de energia. 


\section{Introdução}

As cidades inteligentes estão cada vez mais presentes na gestão e governança das grandes metrópoles. Os avanços no desenvolvimento dos objetos inteligentes [Santos et al. 2016] e seus serviços [Ma et al. 2017] desempenham um papel importante na disseminação do conceito e do progresso das cidades inteligentes. O intuito é fundir informações de objetos e serviços inteligentes para gerenciar, de maneira eficiente, os recursos e serviços de uma grande metrópole [Zanella et al. 2014, Dameri 2017]. Isso tem aumentado o interesse dos pesquisadores em investigar sistemas inteligentes em diferentes domínios de aplicações, tais como smart home, domótica, $e$-health, eficiência energética e ambiente assistido.

Nesse contexto, destacam-se os Sistemas de Automação Residencial (SAR), que podem ser definidos como uma smart home munida com objetos inteligentes e/ou especializados para gerenciar os recursos habitacionais da residência [Cheng and Kunz 2009, Lee et al. 2014, Filho et al. 2015b, Stojkoska and Trivodaliev 2017]. O SAR é uma das aplicações que vêm ganhando destaque nas cidades inteligentes, por ser uma opção promissora para solucionar um dos principais desafios globais, a eficiência energética. Dentro do contexto de SAR, os objetos inteligentes, tais como smart tv, set-top box, roteadores, smart meters e smartphones, podem ser utilizados para monitorar e controlar o ambiente em que estão implantados. Tais objetos podem processar dados e conectar com a Internet, formando um ambiente de fog computing. Ainda, os objetos podem intercomunicar-se para melhorar a qualidade da informação no processo de tomada de decisão [Maia et al. 2015].

O paradigma de fog computing [Bonomi et al. 2014, Mahmud et al. 2017], também conhecido como computação em névoa, surge como uma alternativa promissora para auxiliar objetos inteligentes com recursos computacionais escassos, situação presente nos dispositivos pertencentes a um SAR. Ainda, o paradigma de fog computing pode satisfazer requisitos que não são atendidos por um modelo centralizado. Além disso, tal paradigma estende os recursos computacionais disponíveis na infraestrutura da nuvem para a borda da rede, o que fornece mobilidade, escalabilidade, baixa latência e robustez no serviço aos usuários [Bonomi et al. 2014, Coutinho et al. 2016, Mahmud et al. 2017].

Entretanto, ainda que haja um esforço crescente conquistado na área de SAR, correlacionar dados qualitativos e quantitativos obtidos dos dispositivos para o processo de tomada de decisão (isto é, gerar informações viáveis) dentro da própria infraestrutura de comunicação traz novas questões de pesquisas, das quais duas se destacam, a saber: (i) Como aproveitar os dados que são disseminados de maneira implícita, bruta e incompreensível para prover um aumento no processo de tomada de decisão; e (ii) Como eliminar o problema da interoperabilidade de comunicação e robustez no processamento em dispositivos com recursos escassos para o processo de tomada de decisão.

Com isso em mente, este trabalho vai além das soluções apresentadas por [Soares et al. 2012, Farias et al. 2014, Filho et al. 2015a], propondo o ImPeRIum, um sistema de decisão inteligente que forma um ambiente computacional de fog para gerenciar as aplicações da residência. Nesse caso, foi realizado um estudo preliminar em nosso trabalho anterior [Rocha Filho et al. 2018]. No ImPeRIum, o processo de tomada de decisão é realizado mediante técnicas de inteligência computacional. O paradigma fog computing foi utilizado para integrar uma maior inteligência na infraestrutura de controle da casa. Diante disso, é possível processar o conteúdo localmente na infraestrutura e, 
ganhar tempo na decisão. Tal infraestrutura é responsável por coletar e disseminar os dados no ambiente e, em seguida, detectar e atuar nas aplicações desejadas. Para disseminar os dados no ImPeRIum, implementou-se um módulo de comunicação baseado no paradigma Publish/Subscribe (Pub/Sub) que também permite conectar novos dispositivos e lidar com o problema de interoperabilidade entre eles. Vale salientar, ainda, que para reduzir o overhead de processamento da infraestrutura, as funcionalidades do ImPeRIum são distribuídas entre os nós da rede. Os resultados de uma extensa avaliação, evidenciam a viabilidade e eficiência do ImPeRIum quando comparado com outras soluções da literatura.

O restante deste artigo está organizado da seguinte forma. A Seção 2 apresenta os trabalhos relacionados, discutindo os principais desafios frente a esta pequisa. A Seção 3 descreve como nossa solução foi desenvolvida e a Seção 4 como a solução foi validada. Por fim, a Seção 5 apresenta as conclusões e os trabalhos futuros.

\section{Trabalhos relacionados}

Esta seção discute os principais desafios e problemas em aberto em SAR que ainda não foram solucionados e que esta pesquisa investiga. Por exemplo, até o presente momento, não foi encontrada uma solução que aproveita os dispositivos inteligentes da residência para formar um ambiente computacional de $f o g$, o qual gerencia o processo de tomada de decisão das aplicações do morador.

Atualmente, tem-se investigado o potencial uso das tecnologias de rede sem fio como infraestrutura de comunicação interna de uma residência [Mantoro et al. 2011, Ramlee et al. 2013, Filho et al. 2013, Filho et al. 2015a]. Particularmente em [Mantoro et al. 2011], é proposto um framework que é modelado com base em uma infraestrutura sem fio. Como prova de conceito do framework, foi desenvolvido um protótipo chamado Integ's smart home. O Integ's smart home permite monitorar e controlar as condições da residência através de um dispositivo móvel (por exemplo, smartphone) habilitado para a Internet. Já em [Ramlee et al. 2013], é proposto um sistema de automação residencial de baixo custo voltado para os eletrodomésticos. Uma placa de controle principal (nó sink) é desenvolvida para gerenciar a comunicação entre os eletrodomésticos e os moradores. Entretanto, as soluções citadas anteriormente possuem limitações, diferenciando deste trabalho principalmente por serem desenvolvidas para aplicações específicas, além de não correlacionarem os dados coletados entre as aplicações para melhorar a tomada de decisão.

Em [Mahmood et al. 2014], é proposto o Home Appliances Coordination Scheme for Energy Management (HACS4EM). O HACS4EM tem como objetivo reduzir o custo de energia do morador, deslocando o funcionamento dos eletrodomésticos mediante o valor das tarifas de energia. Para isso, o HACS4EM envolve a comunicação entre os eletrodomésticos e uma unidade de gestão de energia central, a qual é responsável pelo processamento da infraestrutura. Para realizar a comunicação, utilizou-se o protocolo ZigBee para transmitir as mensagens entre as entidades envolvidas no HACS4EM. Portanto, o consumidor pode ligar qualquer eletrodoméstico a qualquer momento e, em seguida, o HACS4EM sugere a hora de uso mais adequada. Entretanto, o HACS4EM diferencia-se desta pesquisa por desencadear uma ação pré-determinada, não aproveitando os dispositivos da casa que podem intercomunicar-se para melhorar a qualidade da informação no 
processo de tomada de decisão.

Outros trabalhos relacionados que merecem destaques são propostos por [Soares et al. 2012, Farias et al. 2014]. Convém salientar que o trabalho apresentado por [Farias et al. 2014], é uma extensão do trabalho realizado anteriormente em [Soares et al. 2012]. Particularmente em [Farias et al. 2014], é proposto um sistema de CONtrole e DEcisão (CONDE) com o intuito de automatizar o processo de tomada de decisão em um ambiente usando uma rede de sensores e atuadores sem fio. O CONDE é desenvolvido para coletar e manipular os dados sensoreados, processando-os dentro da própria infraestrutura. O CONDE utiliza processos de inferências como método para a tomada de decisão. Nesse caso, modelou-se um conjunto de parâmetros e regras para as decisões serem tomadas em cada aplicação ativa na rede de sensores e atuadores sem fio. As limitações do CONDE em relação a este trabalho são: (i) regras pré-estabelecidas para a tomada de decisão; e (ii) há um indicativo de aumento do uso da memória a medida que novas aplicações são inseridas na infraestrutura. A primeira limitação não representa na prática o comportamento do morador, o qual pode alterar o seu comportamento, por exemplo, no final de semana. Já a segunda limitação, além de necessitar de um especialista para gerar as regras, pode haver um problema de escalabilidade em dispositivos com recursos escassos, haja vista a necessidade de criar novas regras de inferência e integração.

O trabalho que mais se assemelha com esta pesquisa é apresentado por [Filho et al. 2015a, Filho et al. 2018]. Os autores [Filho et al. 2015a] propõem um sistema de decisão inteligente para uma infraestrutura residencial distribuída utilizando sensores e atuadores sem fio, chamado ResiDI. O núcleo da inteligência do ResiDI é baseado em uma rede neural que visa melhorar a precisão da tomada de decisão, juntamente com um mecanismo de correlação temporal que é projetado para reduzir o consumo de energia da infraestrutura. Apesar do ResiDI aproveitar o processamento dos nós da rede de sensores sem fio, melhorando a precisão na tomada de decisão, não é capaz de utilizar os dispositivos inteligentes da própria residência para gerenciar as aplicações e, consequentemente, utilizar a intercomunicação de tais dispositivos para melhorar a qualidade da informação no processo de tomada de decisão. Vale frisar, ainda, que existe um problema de interoperabilidade e integração com novos dispositivos. Em outras palavras, possui um comportamento específico, e não genérico, dificultando assim sua adaptação a mudanças no ambiente.

Assim, diferentemente de outros trabalhos da literatura, o ImPeRIum propõe uma generalização das operações e abstração das informações, provendo um mecanismo de decisão e atuação mais eficiente, como apresentado a seguir.

\section{Um sistema de controle neuro-fog para infraestruturas residenciais}

Esta seção apresenta o ImPeRIum, um sistema de decisão inteligente com recursos computacionais distribuídos que forma um ambiente computacional de fog para gerenciar as aplicações da residência. O ImPeRIuM foi desenvolvido com base em técnicas de inteligência computacional para realizar o processo de tomada de decisão, bem como com um conjunto de dispositivos inteligentes da residência (por exemplo, set-top box, tvs inteligentes, medidores e roteadores inteligentes) para formar um ambiente computacional de fog (Figura 1a). Para lidar com a heterogeneidade dos dados e eliminar o problema 
de interoperabilidade dos dispositivos que formam o fog computing, o ImPeRIum implementa um módulo de comunicação Publish/Subscribe ${ }^{1}$ (Pub/Sub). O ImPeRIum tem como objetivos principais prover maior robustez no processamento e aumentar a precisão da tomada de decisão, tendo como objetivos específicos eficiência na entrega dos dados ${ }^{2}$ e alta disponibilidade para o usuário.

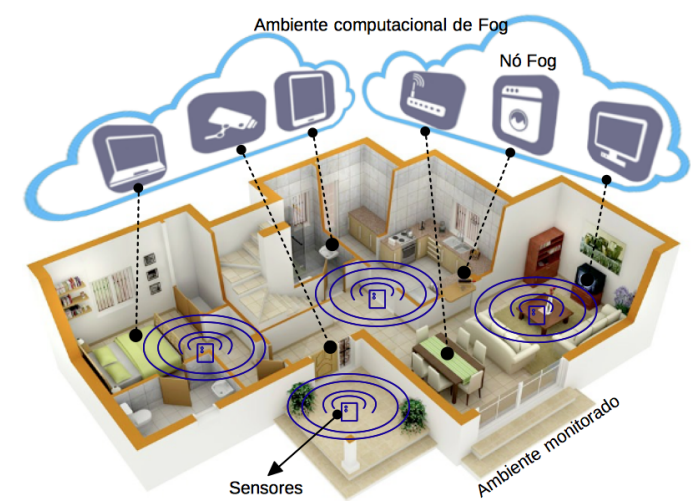

(a) Cenário de funcionamento do ImPeRIum

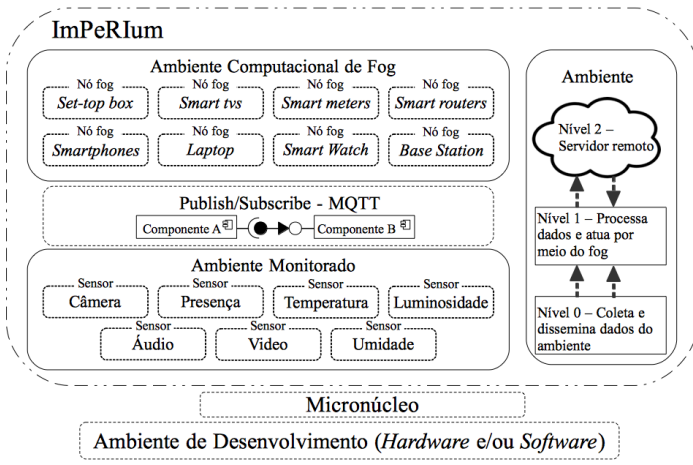

(b) Arquitetura de componentes do ImPeRIum

Figura 1. ImPeRIum - Funcionamento do sistema de decisão inteligente para infraestruturas residenciais

Na solução, assume-se que os nós são munidos com uma interface de comunicação sem fio, armazenamento, processamento e bateria como fonte de backup. A Figura 1b ilustra a arquitetura de componentes do ImPeRIum, no qual os nós publicam (publish) seus dados e, em seguida, os nós assinantes (subscribe) recebem os dados correspondentes para processar e retornar uma resposta. A arquitetura do sistema além de ser dividida em papéis ${ }^{3}$, também é distribuída em níveis: (i) Nível 0 , sensores utilizados para coletar os dados do ambiente e disseminar tais dados para o Nível 1; (ii) Nível 1, dispositivos embarcados que além de processar os dados, também podem monitorar e controlar o ambiente; e (iii) Nível 2, servidor remoto com maior poder de processamento e armazenamento.

O ImPeRIum além de prover uma infraestrutura com alta disponibilidade caso um dispositivo falhe, ele aumenta a cobertura no gerenciamento do ambiente. Além disso, a arquitetura proposta maximiza a eficiência na entrega dos dados com uma baixa latência, uma vez que os dispositivos estão localizados mais próximos da região de interesse e o processo de tomada de decisão, bem como a sua atuação é realizada no próprio dispositivo (isto é, no ambiente de fog computing). De outro ponto de vista, uma abordagem tradicional que utiliza um sink para realizar o gerenciamento do ambiente, caso o sink falhe, toda a rede fica inoperante. Ainda nesse cenário, caso o sink fosse considerado como um atuador, haveria um consumo desigual dos recursos devido ao fluxo de dados próximos ao sink ser mais intenso. Destaca-se, também, que caso ocorra uma falha no componente de atuação do sink a tarefa não é finalizada com êxito, isto é, mesmo o sink

\footnotetext{
${ }^{1}$ Com o Publish/Subscribe pode-se criar grupos de interesse e a entrega das mensagens é direcionada a tais grupos, o que faz com que somente os interessados sejam envolvidos no processo de transmissão e, portanto acarretando em menor consumo energético.

${ }^{2} \mathrm{O}$ sistema deve ser capaz de disseminar os dados com baixo atraso e com um número menor de transmissões, maximizando a energia residual dos dispositivos.

${ }^{3}$ Entende-se como papéis as funcionalidades específicas que são atribuídas para cada nó
} 
funcionando a rede ficaria inoperante para realizar as atuações no ambiente. Para um melhor entendimento da solução, o ImPeRIum é detalhado em três etapas, sendo elas: (i) divisão dos papéis com a descrição do seu funcionamento; (ii) comunicação do sistema; e (iii) inteligência computacional para o processo de tomada de decisão. Cada uma dessas etapas são descritas nas subseções seguinte.

\subsection{Funcionamento dos papéis e níveis do ImPeRIum}

Para monitorar e gerenciar o ambiente, os papéis são distribuídos entre os nós (revisite a Figura 1) da rede, sendo eles: (i) nó sensor; e (ii) nó fog. Os nós sensores (Nível 0) são distribuídos no ambiente para coletar e disseminar dados, transmitindo-os para o ambiente computacional de fog (Nível 1). O ambiente computacional de fog é composto por dispositivos que estão próximo da borda da rede com poder de processamento, armazenamento e de comunicação entre os usuários finais e os servidores de nuvem tradicionais (Nível 2) [Mahmud et al. 2017]. No ImPeRIum, tais dispositivos são chamados de nós fogs, sendo responsáveis por receber os dados dos sensores e realizar o processamento para detectar a tomada de decisão e atuar no ambiente. Nesse caso, as informações para realizar a decisão são processadas no próprio nó fog. A implementação do modelo de tomada de decisão (descrita na Seção 3.3) para o ambiente de fog foi realizada no Nível 2. O ambiente computacional de fog é responsável por toda região de detecção e atuação. Para isso, o ImPeRIum possui um Mapa Virtual dos Fogs (MVF). Com isso, cada nó fog possui seu conhecimento local e de todo o ambiente. Para adquirir o conhecimento de todo o ambiente, os nós fogs compartilham dados entre si por meio de um modelo request-reply via $P u b / S u b$. Em relação a atuação, o nó fog controla as aplicações (iluminação e equipamentos eletrodomésticos) do ambiente por meio de diferentes componentes de atuação. Isso é uma realidade razoável de supor, uma vez que os dispositivos inteligentes podem possuir diferentes tipos de atuadores. O processo para realizar a comunicação do ImPeRIum é descrito na próxima Subseção.

\subsection{Comunicação Publish/Subscribe do ImPeRIum}

No ImPeRIum, o paradigma Pub/Sub [Eugster et al. 2003] permite a disseminação confiável do conteúdo de maneira assíncrona entre os fornecedores (Publish) e os consumidores (Subscribe) de informações. Para a comunicação entre os nós, baseada no paradigma Pub/Sub, foi utilizado o protocolo de comunicação Message Queue Telemetry Transport (MQTT) [Stanford-Clark and Nipper 2014] (isto é, barramento Publish/Subscribe - MQTT, Figura 2(a)) que está implementado em todos os nós do ImPeRIum. O barramento gerencia os fornecedores de conteúdo e seus assinantes, de maneira que, tal barramento filtra os eventos recebidos e os repassam para os assinantes de acordo com o conteúdo da assinatura, como ilustrado na Figura 2(a). Em outras palavas, um determinado nó publica seus dados e, em seguida, os nós assinantes recebem os dados correspondentes para processar e retornar uma resposta. Assim, é possível garantir a entrega confiável dos dados publicados pelos nós para os componentes do ambiente de execução do ImPeRIum.

O processo de publicação ocorre quando o nó sensor $\left(S_{n}\right)$ gera um evento (Rótulo A, Figura 2(b)). Neste caso, o $S_{n}$ cria uma mensagem de publicação contendo o seu ID e o conteúdo do evento. Antes de enviar a mensagem (Rótulo B, Figura 2(b)), o ImPeRIum utiliza o protocolo de correlação temporal [Filho et al. 2015a]. Tal protocolo correlaciona 


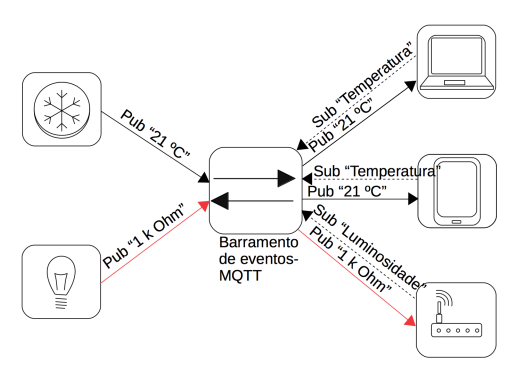

(a) Barramento de eventos.

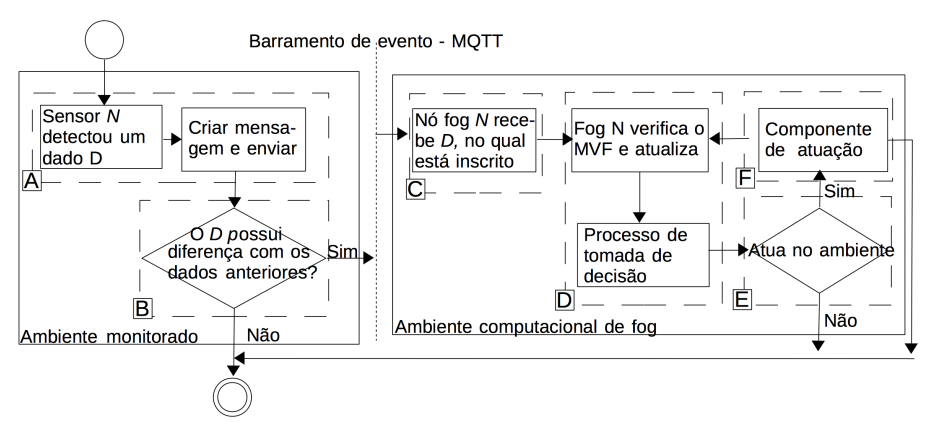

(b) Fluxograma de comunicação do ImPeRIum.

Figura 2. ImPeRIum - Comunicação Pub/Sub por meio do MQTT.

os eventos gerados ao tempo, comparando o conteúdo do evento atual com os conteúdos dos eventos anteriores para publicar, ou não, o evento gerado. Com isso, o ImPeRIum consegue eliminar as notificações redundantes, aumentando o tempo de vida da rede e reduzindo a quantidade de comunicação entre os nós. Após o $S_{n}$ publicar o conteúdo, o nó fog $\left(F_{n}\right)$ que está inscrito naquele conteúdo recebe o evento publicado (Rótulo $\mathrm{C}$, Figura 2(b)). Para o $F_{n}$ receber o conteúdo, é necessário inscrever-se no barramento de evento com uma mensagem contendo o seu ID, o conteúdo a ser recebido e os tipos de aplicações que irá gerenciar. Ainda, o $F_{n}$ também se registra em outros nós fogs para receber dados de outros sensores, os quais não está em seu alcance. Tais dados são armazenados no MVF e, dessa forma, o ImPeRIum adquire o conhecimento local e de todo ambiente para realizar o processo de tomada de decisão (Rótulo D, Figura 2(b)). Tal processo é iniciado quando o $F_{n}$ recebe os dados dos sensores. Assim, por meio de uma rede neural, o $F_{n}$ verifica se é necessário atuar no ambiente (Rótulo E, Figura 2(b)). Com isso, a informação da aplicação e sua respectiva atuação são adquiridas com o intuito de atuar no ambiente (Rótulo F, Figura 2(b)). Tal informação é armazenada no MVF para evitar conflitos de atuação ou interesse ou caso outro $F_{n+1}$ deseja atuar no ambiente. $\mathrm{O}$ processo para a tomada de decisão é descrito a seguir.

\subsection{Inteligência computacional para o processo de tomada de decisão}

Para realizar o processo de tomada de decisão, foram explorados seis algoritmos de classificação clássicos da literatura, sendo eles: (i) Lógica fuzzy; (ii) Árvore de Decisão (iii) K-Nearest Neighbors, KNN; (iv) Naive Bayes; (v) Rede Neural Artificial do tipo MultiLayer Perceptron, RNA; e (vi) Comitê de classificadores. Os algoritmos utilizam os dados que estão no MVF e, em seguida, detectam as características do ambiente de acordo com a preferência do morador para realizar o processo de tomada de decisão.

Para saber qual algoritmo utilizar na modelagem do ImPeRIum, realizou-se uma análise exploratória, como ilustrado na Figura 3. Para obter os modelos de classificação, utilizou-se os dados obtidos em [Bache and Lichman 2013, Filho et al. 2014] que foram aplicados em [Filho et al. 2015a]. A Figura 3(a) apresenta a porcentagem dos resultados relativos à métrica precisão em função dos algoritmos de classificação. Já a Figura 3(b) fornece um ranking que permite determinar o desempenho geral de cada um dos algoritmos avaliados e suas diferenças estatísticas.

Com base nesses resultados, nota-se que o Comitê e a RNA obtiveram os melhores resultados. Apesar do Comitê possuir uma melhor colocação no ranking, o mesmo utiliza 


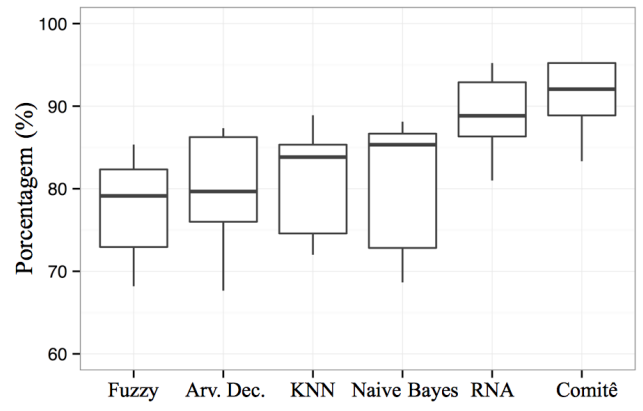

(a) Precisão dos algoritmos de classificação

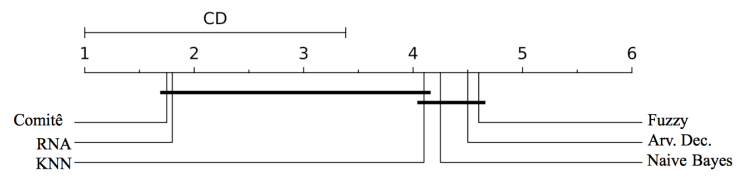

(b) Ranking dos algoritmos de classificação

Figura 3. Análise exploratória dos algoritmos de classificação

vários algoritmos que aumentam significativamente o seu custo computacional. Em razão disso, ao utilizar o Comitê, o processo de tomada de decisão fica mais lento no que diz respeito ao tempo de resposta e processamento. Por outro lado, a RNA possui um menor custo computacional, além de apresentar uma alta taxa de acertos com uma melhor estabilidade nos resultados. Pelos motivos supracitados, a RNA foi utilizada para realizar o processo de tomada de decisão.

Deve-se destacar que a topologia da rede neural adotada na nossa proposta é composta por três camadas (Figura 4), sendo a camada de entrada com sete neurônios responsável por inserir os dados na rede, a camada escondida com quinze neurônios responsável por determinar padrões de regiões de aprendizado, e a camada de saída com três neurônios responsável por fornecer o resultado dos dados inseridos. Vale salientar, ainda, que os dados da camada de saída foram normalizados e a função de ativação adotada na camada escondida foi a sigmóide logística. Além disso, o algoritmo backpropagation foi utilizado para treinar a rede. A rede foi treinada no nível 2 do ImPeRIum e, em seguida disseminada para os nós fogs. Com isso, foi possível reduzir a sobrecarga de processamento no nosso sistema.

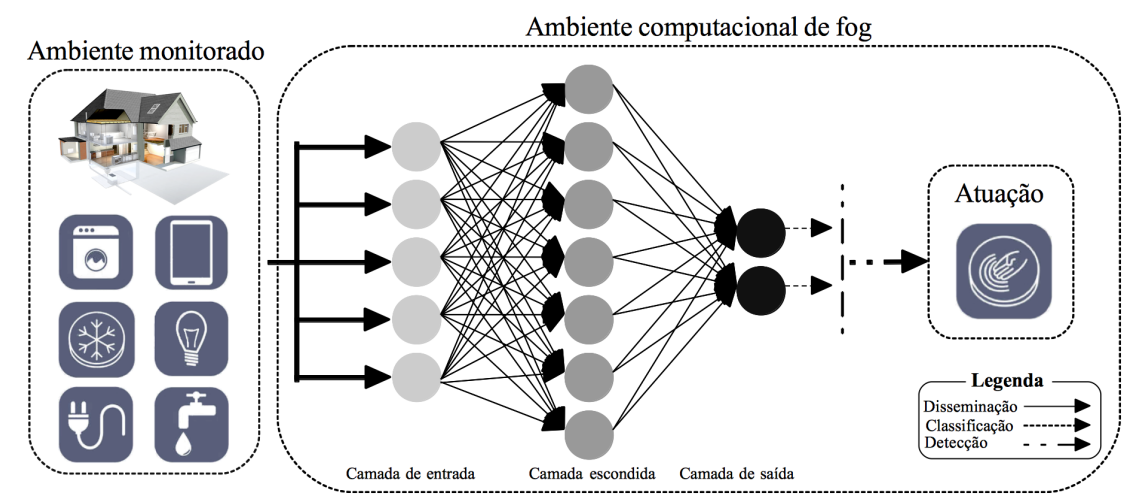

Figura 4. Funcionamento da RNA modelada para o processo de tomada de decisão.

\section{Avaliação de Desempenho e Metodologia}

Nesta seção, são apresentados os resultados da avaliação de desempenho e a metodologia utilizada para gerar os resultados. Para isso, a validação do ImPeRIum foi dividida em dois estágios. No primeiro estágio foi realizada uma validação do ImPeRIum, 
comparando-o com duas abordagens da literatura, CONDE e ResiDI. Tais abordagens foram escolhidas por serem os trabalhos que mais se assemelham com esta pesquisa, como discutido na Seção 2. Já no estágio 2, foi avaliada a gerência de recurso do ImPeRIum em um ambiente de fog, comparando-o com uma abordagem tradicional que utiliza um ambiente de servidor em nuvem.

\subsection{Avaliação de desempenho do ImPeRIum com outras soluções da literatura}

Esta subseção valida o desempenho do ImPeRIum por meio de simulações utilizando o Network Simulator $3^{4}$. Em relação a topologia da rede, adotou-se uma área monitorada de 40x40 m para depositar os nós. Para cobrir toda área de coleta e disseminação de dados da topologia, a densidade dos sensores é de 0.015 nós $/ \mathrm{m}^{2}$. Já a densidade dos nós fogs é de 0.007 nós $/ \mathrm{m}^{2}$, uma vez que é realístico admitir que há menos nós processando dados que sensoreando o ambiente. O raio de comunicação dos nós é de $15 \mathrm{~m}$, e o seu consumo de energia para transmitir e receber um pacote são de $0,2088 \mu \mathrm{J} / \mathrm{bit}$ e $0,2256 \mu \mathrm{J} / \mathrm{bit}$, respectivamente, de acordo com a plataforma MicaZ.

No ImPeRIum, foram implementadas duas aplicações residenciais: (i) controle do sistema de iluminação residencial; e (ii) controle energético dos eletrodomésticos, especificamente televisão, computador e geladeira. No caso do sistema de iluminação, três tipos de intensidade luminosa (baixa, média e alta) foram consideradas para atuação. Já no sistema de eletrodoméstico, considerou-se o estado ligar/standy/desligar para atuação. Para obter um cenário mais próximo de um ambiente real, utilizou-se como entrada para a simulação os conjuntos de dados reais obtidos em [Bache and Lichman 2013, Filho et al. 2014] com os seguintes atributos: (i) temperatura do ambiente em grau Celsius; (ii) presença de pessoas no ambiente; (iii) data e horário de utilização da aplicação; (iv) potência em Watt da aplicação; (v) estado da aplicação; (vi) tipo de aplicação; e (vii) tomada de decisão. Tais atributos foram transformados em blocos de dados (Figura 5) para simular a disseminação de vários eventos na residência. Destaca-se, também, que as aplicações implementadas possuem diferentes intensidades de uso durante o dia, como apresentada na Figura 6.
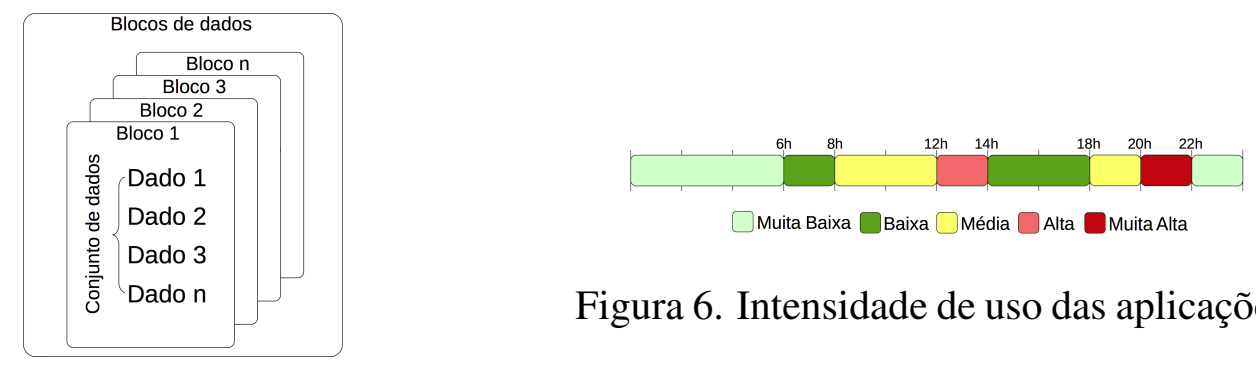

Figura 6. Intensidade de uso das aplicações.

Figura 5. Bloco de dados utilizados.

Para avaliar o ImPeRIum, utilizou-se as métricas: (i) taxa de acerto, representa a porcentagem de acertos classificados corretamente no processo de tomada de decisão; (ii) tempo de resposta, representa o tempo gasto desde o sensoriamento até a atuação para finalizar a execução de uma decisão; (iii) transmissão de dados, representa o total de mensagens transmitidas na infraestrutura durante o processo de tomada de decisão; e (iv) consumo de energia, representa o total de energia consumida pela infraestrutura no

\footnotetext{
${ }^{4}$ Network Simulator 3 - NS3, http://www.nsnam.org/
} 
processo de tomada de decisão. Os experimentos foram executados 33 vezes com um intervalo de confiança de $95 \%$ de acordo com a distribuição $t$-student. Os resultados são apresentados na próxima subseção.

\subsubsection{Análise dos resultados obtidos}

Na Figura 7(a), é apresentada a taxa de acerto no processo de tomada de decisão variando a quantidade de bloco de dados $(8,16,32,64$ e 128). Por meio dos resultados, notou-se que o ImPeRIum possui em média 1.17 vezes mais acertos que o CONDE. Isso ocorre pois o CONDE modelou a tomada de decisão por meio de tecnologias semáticas (isto é, parâmetros e regras), diferentemente do ImPeRIum que generaliza as tomadas de decisões baseado em exemplos históricos. Com isso, o ImPeRIum realiza o acerto das tomadas de decisões para exemplos nunca vistos. Quando comparado com o ResiDI, o ImPeRIum foi estatisticamente equivalente independente da quantidade de bloco de dados publicado na rede. Isso é coerente, posto que ambas soluções utilizam o mesmo conceito de aprendizagem para o processo de tomada de decisão. Além disso, a similaridade entre o ImPeRIum e o ResiDI ratifica a eficiência da inteligência computacional na tomada de decisão.

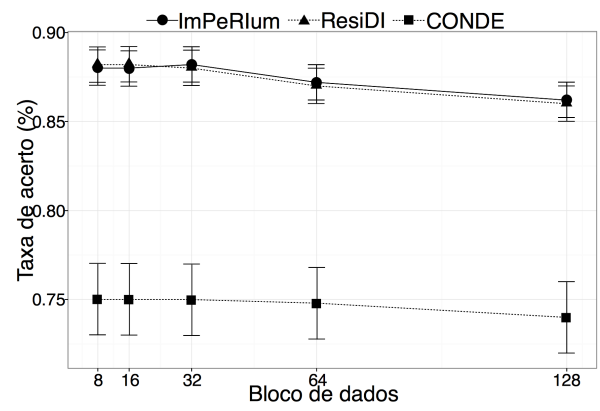

(a) Taxa de acerto

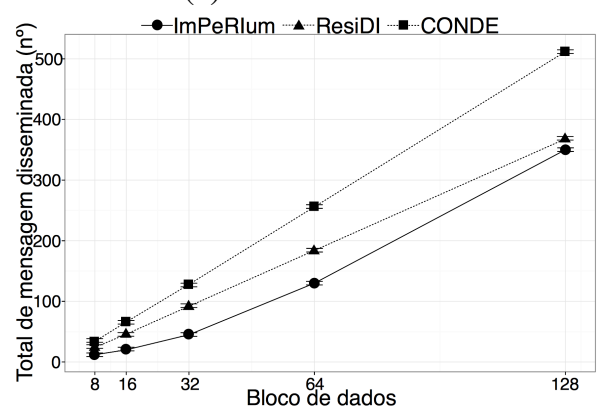

(c) Transmissão de dados

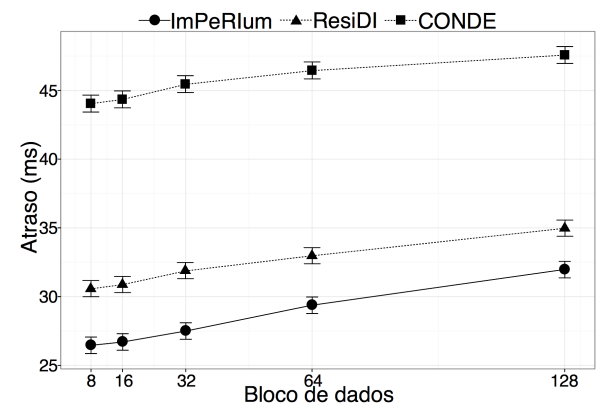

(b) Tempo de resposta

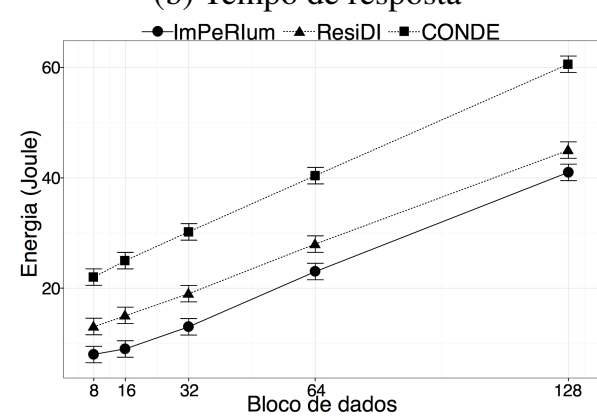

(d) Consumo de energia

Figura 7. Impacto de desempenho do ImPeRIum quando comparado com outras abordagens da literatura.

Após validar a taxa de acertos, foi analisado o tempo gasto para que uma decisão seja executada, como apresentado na Figura 7(b). Como pode ser observado, o ImPeRIum possui um tempo de resposta menor quando comparado com o ResiDI e o CONDE. Em média o ImPeRIum foi $13 \%$ e $60 \%$ mais rápido que o ResiDI e CONDE, respectivamente. No pior caso, 128 blocos de dados, o ImPeRIum foi 9\% mais rápido que o ResiDI e 48\% mais rápido que o CONDE. Isto está relacionado com o ambiente computacional de fog, no qual não necessita transmitir a informação da atuação para um dispositivo específico. 
Em relação a métrica total de mensagem disseminada, Figura 7(c), observou-se que o ImPeRIum dissemina em média 1.74 e 2.45 vezes menos dados quando comparado com o ResiDI e o CONDE, respectivamente. Isso reduz o número de colisões na rede e preserva a energia residual do dispositivo. Isso ocorre pois, a atuação é realizada no próprio ambiente computacional de fog. Isto é, não é necessário repassar as informações da atuação para outro dispositivo na rede e, consequentemente, há menos mensagens disseminadas. Portanto, o ImPeRIum possui menos sobrecarga na disseminação de dados que as demais soluções.

Por fim, a Figura 7(d) apresenta a métrica consumo de energia dos dispositivos em função da quantidade de bloco de dados. Em todos os cenários avaliados, observa-se que o ImPeRIum consome menos energia residual dos dispositivos à medida que a quantidade de bloco de dados aumenta. Isso é uma consequência não apenas da redução da quantidade de mensagem transmitida, mas também é devido a robustez no processamento para a tomada de decisão por meio do ambiente computacional de fog, como pode ser revisto nas figuras 7(b) e 7(c). O pior caso ocorre com 128 blocos de dados, sendo que o ImPeRIum possui uma redução de $9 \%$ no consumo de energia em relação ao ResiDI e $47 \%$ no consumo de energia em relação ao CONDE. Portanto, o ImPeRIum é capaz de preservar a energia residual dos dispositivos, mantendo uma precisão na tomada de decisão, além de garantir robustez no processamento (isto é, tempo de resposta menor) com uma redução na transmissão de dados.

\subsection{Avaliação de desempenho do ImPeRIum no ambiente de fog}

Nesta subseção, é avaliada a gerência de recurso do ImPeRIum, apresentado a sua eficiência em um ambiente computacional de fog, comparando-o com uma abordagem tradicional que utiliza um ambiente de servidor em nuvem. Os experimentos foram executados em diferentes ambientes reais, chamados de níveis: (i) nível 1, ambiente computacional de fog; e (ii) nível 2, servidor remoto. Para cada nível foram utilizados os blocos de dados e as aplicações apresentadas na Subseção 4.1. A descrição do conjunto de parâmetros estabelecidos para realizar a avaliação e os dispositivos físicos utilizados para os experimentos são apresentados na Tabela 1. Como o objetivo da avaliação é determinar a eficiência da gerência de recurso do ImPeRIum em um ambiente de fog, utilizou-se como variável de resposta as métricas consumo de memória, consumo de processador e tempo de processamento das aplicações. Destaca-se que o tempo de processamento das aplicações, considera o tempo gasto entre o envio dos dados e a resposta da ação. Os experimentos foram replicados 33 vezes com uma confiança de $95 \%$ de acordo com a distribuição t-student. Os resultados de tais experimentos são apresentados a seguir.

Tabela 1. Conjunto de parâmetros para serem avaliados e os dispositivos físicos utilizados nos experimentos.

\begin{tabular}{|c|c|c|c|c|c|c|}
\hline \multicolumn{7}{|c|}{ Descrição dos parâmetros } \\
\hline \multirow{2}{*}{ Experimento usando o nó fog e servidor remoto } & Nó fog $^{1}$ & \multicolumn{5}{|c|}{ Servidor remoto } \\
\hline & $E 1^{1}$ & E2 & E3 & E4 & E5 & E6 \\
\hline Quantidade de blocos de dados & $128^{1}$ & 8 & 16 & 32 & 64 & 128 \\
\hline \multicolumn{7}{|c|}{\begin{tabular}{|c|} 
Descrição dos dispositivos físicos \\
\end{tabular}} \\
\hline Nó fog - Raspberry Pi 2 & \multicolumn{6}{|c|}{ ARMV7-A, BCM2836@900 MHz, 1GB } \\
\hline Servidor remoto - Computador & \multicolumn{6}{|c|}{ Intel Core I7-7700K, @4.2 GHz, 16GB } \\
\hline
\end{tabular}




\subsubsection{Análise dos resultados obtidos}

Inicialmente, foi avaliado a gerência de recursos do ImPeRIuM por meio das métricas consumo de memória e processador em função da quantidade de dados no momento da tomada de decisão. Nota-se que há uma linearidade de ambas as métricas (Figuras 8(a) e 8(b)), independente dos níveis e dos blocos de dados de entrada. Um aspecto importante, notado nas Figuras 8(a) e 8(b), está relacionado com os níveis. Em particular, o nível 1 possui maior consumo de memória e processador quando comparado com as variações do nível 2. Entretanto, tal consumo não é grave, não ultrapassando $3 \%$ de memória e $45 \%$ de processador. Consequentemente, o nível 1 possui ciclos ociosos de processador e suporta a execução de aplicações sem sobrecarregar os dispositivos. Com isso em mente, é possível aproveitar os recursos computacionais dos dispositivos que estão na borda da rede para coletar e atuar no ambiente mediante ofog computing.

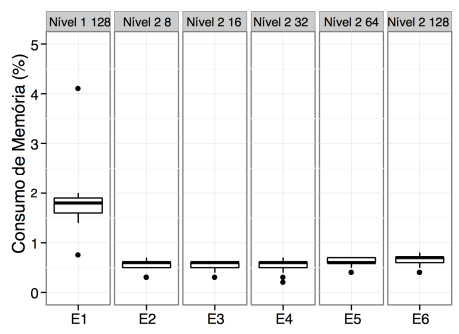

(a) Desempenho do consumo de memória

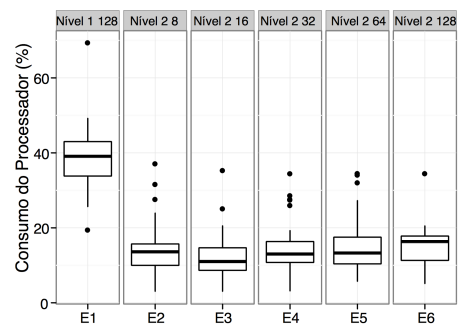

(b) Desempenho do consumo de processamento

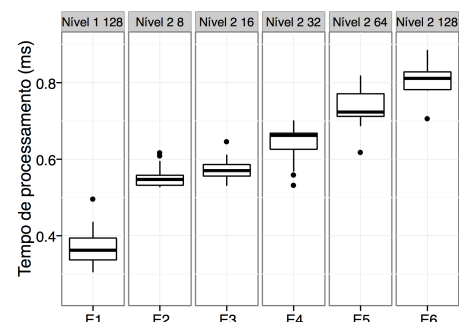

(c) Desempenho do tempo de processamento

Figura 8. Impacto de desempenho do consumo de memória, processador e tempo de processamento

Após notar os ciclos ociosos de processamento do nível 1 sem sobrecarregá-lo, foi avaliado o tempo de processamento da aplicação, como pode ser observado na Figura 8(c). Nota-se que o tempo de processamento do ambiente computacional de fog com 128 blocos de dados é 2.1 vezes, no melhor caso (Experimento E6, Figura 8(c)), e 1.4 vezes, no pior caso (Experimento E2, Figura 8(c)), mais rápido que o ambiente em nuvem para realizar o processo da tomada de decisão. Isso ocorre devido ao ambiente computacional de fog que, além de possuir recursos disponíveis para realizar o processamento, não necessita repassar os dados para um ambiente externo (isto é, nível 2). Portanto, tais afirmações evidenciam a eficiência do ImPeRIum em um ambiente computacional de fog, apresentado robustez no processamento das tomadas de decisões, sem sobrecarregar os recursos do sistema.

\section{Conclusão}

Este artigo propôs o ImPeRIum, uma abordagem inteligente para um ambiente residencial que, além de manter uma precisão no processo de tomada de decisão, tem como objetivo melhorar a robustez no processamento, reduzindo a latência de serviço em dispositivos com recursos escassos. Uma extensa avaliação evidenciou a viabilidade do ImPeRIum em ambientes inteligentes com dispositivos de recursos escassos. Os resultados obtidos dessa avaliação mostraram ser promissores, sendo três deles notáveis: (i) alta taxa de acertos com baixo atraso no processo de tomada de decisão; (ii) eficiência na disseminação da informação com baixo overhead na infraestrutura; e (iii) robustez no processamento com baixo consumo de energia. 
Como trabalhos futuros, planeja-se além de ampliar o estudo apresentado neste artigo, aplicar a solução proposta em diferentes contextos, tais como predial, segurança e health smart homes. Além disso, desenvolver mecanismos de consenso para que as decisões entre os nós fogs sejam correlacionadas com os diversos ambientes da residência.

Agradecimentos: Geraldo P. R. Filho, Leandro A. Villas e Jó Ueyama agradecem à FAPESP (processos 2014/06330-5, 2015/24494-8 e 2015/21642-6) por financiarem seus projetos de pesquisas. Por fim, Rodolfo I. Meneguette e Jó Ueyama agradecem à CNPq (processos 150545/2018-5 e 304530/2016-6) por financiarem seus projetos de pesquisas.

\section{Referências}

Bache, K. and Lichman, M. (2013). UCI machine learning repository. Irvine, CA: University of California, School of Information and Computer Science.

Bonomi, F., Milito, R., Natarajan, P., and Zhu, J. (2014). Fog Computing: A Platform for Internet of Things and Analytics, pages 169-186. Springer International Publishing, Cham.

Cheng, J. and Kunz, T. (2009). A survey on smart home networking. Carleton University, Systems and Computer Engineering.

Coutinho, A. A. T. R., Carneiro, E. O., and Greve, F. G. P. (2016). Computação em névoa: Conceitos, aplicações e desafios. pages $266-315$.

Dameri, R. P. (2017). Using ICT in Smart City, pages 45-65. Springer International Publishing, Cham.

Eugster, P. T., Felber, P. A., Guerraoui, R., and Kermarrec, A.-M. (2003). The many faces of publish/subscribe. ACM Comput. Surv., 35(2):114-131.

Farias, C., Soares, H., Pirmez, L., Delicato, F., Santos, I., Carmo, L. F., Souza, J., Zomaya, A., and Dohler, M. (2014). A control and decision system for smart buildings using wireless sensor and actuator networks. Transactions on Emerging Telecommunications Technologies, 25(1):120-135.

Filho, G., Ueyama, J., Faical, B., Guidoni, D., and Villas, L. (2015a). Residi: An intelligent decision platform for residential infrastructure using wireless sensors and actuators. In Computer Networks and Distributed Systems (SBRC), 2015 XXXIII Brazilian Symposium on, pages 237-245.

Filho, G. P. R., Ueyama, J., Faical, B. S., Pessin, G., d. Farias, C. M., Pazzi, R. W., Guidoni, D. L., and Villas, L. A. (2015b). An energy-aware system for decisionmaking in a residential infrastructure using wireless sensors and actuators. In Network Computing and Applications (NCA), 2015 IEEE 14th International Symposium on, pages 9-16.

Filho, G. P. R., Ueyama, J., Villas, L., Pinto, A., Goncalves, V. P., and Seraphini, S. (2013). An intelligent approach for improving energy efficiently in smart grids. In International Conference on Wireless Networks (ICWN), volume 12, pages 227-233.

Filho, G. P. R., Ueyama, J., Villas, L. A., Pinto, A. R., Goncalves, V. P., Pessin, G., Pazzi, R. W., and Braun, T. (2014). Nodepm: A remote monitoring alert system for energy consumption using probabilistic techniques. Sensors, 14(1):848. 
Filho, G. P. R., Villas, L. A., Freitas, H., Valejo, A., Guidoni, D. L., and Ueyama, J. (2018). Residi: Towards a smarter smart home system for decision-making using wireless sensors and actuators. Computer Networks, 135:54 - 69.

Lee, J. H., Lee, H., Kim, M. J., Wang, X., and Love, P. E. (2014). Context-aware inference in ubiquitous residential environments. Computers in Industry, 65(1):148 - 157.

Ma, M., Preum, S. M., and Stankovic, J. A. (2017). Cityguard: A watchdog for safetyaware conflict detection in smart cities. In Internet-of-Things Design and Implementation (IoTDI), 2017 IEEE/ACM Second International Conference on, pages 259-270. IEEE.

Mahmood, A., Khan, I., Razzaq, S., Najam, Z., Khan, N., Rehman, M., and Javaid, N. (2014). Home appliances coordination scheme for energy management (hacs4em) using wireless sensor networks in smart grids. In Procedia Computer Science, volume 32, pages 469 - 476. Elsevier.

Mahmud, R., Kotagiri, R., and Buyya, R. (2017). Fog Computing: A Taxonomy, Survey and Future Directions, pages 103-130. Springer Singapore, Singapore.

Maia, P., Baffa, A., Cavalcante, E., Delicato, F. C., Batista, T., and Pires, P. F. (2015). Uma plataforma de middleware para integração de dispositivos e desenvolvimento de aplicações em e-health. pages $361-374$.

Mantoro, T., Ayu, M. A., and Elnour, E. E. (2011). Web-enabled smart home using wireless node infrastructure. In Proceedings of the 9th International Conference on Advances in Mobile Computing and Multimedia, MoMM'11, pages 72-79, New York, NY, USA. ACM.

Ramlee, R., Othman, M., Leong, M., Ismail, M., and Ranjit, S. (2013). Smart home system using android application. In Information and Communication Technology (ICoICT), 2013 International Conference of, pages 277-280, Bandung. IEEE.

Rocha Filho, G. P., Mano, L. Y., Valejo, A. D. B., Villas, L. A., and Ueyama, J. (2018). A low-cost smart home automation to enhance decision-making based on fog computing and computational intelligence. IEEE Latin America Transactions, 16(1):186-191.

Santos, B. P., Silva, L., Celes, C., Borges, J. B., Neto, B. S. P., Vieira, M. A. M., Vieira, L. F. M., Goussevskaia, O. N., and Loureiro, A. (2016). Internet das coisas: da teoriaa prática. Minicursos SBRC-Simpósio Brasileiro de Redes de Computadores e Sistemas Distribuidos.

Soares, H., Pirmez, L., Delicato, F., and de Farias, C. (2012). Conde: Um sistema de controle e decisao para edificios inteligentes usando redes de sensores e atuadores sem fio. In XXX Simposio Brasileiro de Redes de Computadores e Sistemas Distribuidos (SBRC), pages $117-130$.

Stanford-Clark, A. and Nipper, A. (2014). Mq telemetry transport.

Stojkoska, B. L. R. and Trivodaliev, K. V. (2017). A review of internet of things for smart home: Challenges and solutions. Journal of Cleaner Production, 140, Part 3:1454 1464.

Zanella, A., Bui, N., Castellani, A., Vangelista, L., and Zorzi, M. (2014). Internet of things for smart cities. IEEE Internet of Things Journal, 1(1):22-32. 